\title{
Nutzenbewertung der DMP Diabetes mellitus
}

\section{Neue Erkenntnisse aus dem Vergleich von DMP-Teilnehmern und Nichtteilnehmern anhand von GKV-Routinedaten und einer Patientenbefragung}

Fünf Jahre nach Einführung der Disease Management Programme (DMP) in der GKV wird die Frage nach deren Nutzen immer wichtiger. Trotz zahlreicher Qualitätsberichte, Patientenbefragungen und ersten Ergebnissen der gesetzlich vorgeschriebenen Programmevaluation bleiben jedoch Zweifel, ob Programmeffekte mit den genannten Mitteln überhaupt belegt werden können. Insbesondere fehlen Datenvergleiche von Versicherten, die an einem DMP teilnehmen und Nichtteilnehmern. Eine aktuelle Datenanalyse der BARMER erbringt hierzu neue Erkenntnisse. Es zeigen sich deutliche Unterschiede in der Versorgungssituation zugunsten der am DMP teilnehmenden Diabetiker. Auch nach einer vergleichenden Betrachtung der Ergebnisse einer Patientenbefragung zu diesem Thema ${ }^{2}$ lässt sich dabei die These, bei DMP-Teilnehmern handele sich einfach um eine "Risikoselektion weniger stark betroffener Diabetiker" nicht bestätigen. Zwar sind Selektionseffekte hinsichtlich des Informationsverhaltens der Patienten und zwischen aktiv einschreibenden und nichteinschreibenden Ärzten zu vermuten. Bei der objektiven Bewertung "echter" Programmeffekte müssen aber diese systematischen Versorgungsunterschiede, die letztlich beispielhaft für die Potenziale einer flächendeckenden strukturierten Behandlung chronisch Kranker sind, in jedem Fall als bedeutsam eingeschätzt werden

\section{Christian Graf, Walter Ullrich, Ursula Marschall}

BARMER Ersatzkasse, Hauptverwaltung Wuppertal

\section{Hintergrund}

Seit dem Jahr 2002 werden Disease Management Programme (DMP) in der gesetzlichen Krankenversicherung (GKV) sukzessive eingeführt. Aktuell nehmen knapp 4 Mio. Versicherte bundesweit an einem DMP für die Krankheitsbilder Diabetes mellitus Typ 1 und Typ 2, Koronare Herzkrankheit (KHK), Brustkrebs, Asthma oder COPD teil. Der größte Anteil entfällt mit über 2,5 Mio. Versicherten auf das DMP Diabetes mellitus Typ 2. Damit zählt dieses Programm zu den weltweit größten DMP und findet mittlerweile auch in der internationalen Diskussion Beachtung ${ }^{3}$. Die hohe Beteiligung kann unter anderem auf den starken Anreiz zur Einschreibung für die Krankenkassen aufgrund der Koppelung an den Risikostrukturausgleich zurückgeführt werden. Zugleich wird diese Koppelung häufig für anfängliche Probleme und Fehlentwicklungen in den DMP verantwortlich gemacht.

Dr. Christian Graf, Walter Ullrich, Dr. Ursula Marschall, BARMER Ersatzkasse, Hauptverwaltung Wuppertal
Von Beginn an wurden die DMP von zahlreichen, oftmals emotional geführten Kontroversen begleitet. Im Zentrum des Interesses standen dabei u.a. die Orientierung an evidenzbasierten Leitlinien, der Grad der „Einmischung" in die ärztliche Versorgung durch Krankenkassen und der Verwaltungsaufwand für die Dokumentation und Qualitätssicherung in Arztpraxen ${ }^{4}$. Allen Unkenrufen zum Trotz scheinen diese Probleme nunmehr weitgehend überwunden. Mittlerweile häufen sich Beiträge und Stimmen aus den Reihen der Ärzteschaft, die einen effektiven Nutzen für das Qualitätsmanagement der Praxis und die Patientenorientierung, bei minimalem Zusatzaufwand bestätigen ${ }^{5}$.

Eine weitere, eher akademische Kontroverse bezieht sich auf die Frage, ob mit den bestehenden DMP eine Zielgenauigkeit im Hinblick auf diejenigen Versicherten mit dem höchsten Risiko und somit dem höchsten Optimierungspotential erreicht werden könne ${ }^{6}$. Andererseits wird gerade in der internationalen Debatte häufig darauf hingewiesen, wie bedeutsam ein bevölkerungsbezogener Ansatz über alle Risikogruppen hinweg mit Hausarztori- 
entierung für den Erfolg von DMP ist ${ }^{7}$. Während international aus Sicht der Ärzteschaft zu häufig Disease Management Programme „an den eigentlichen Versorgern“ vorbei implementiert werden, kann die Fokussierung auf den koordinierenden Hausarzt in den deutschen DMP mit gleichzeitiger sekundär präventiver Ausrichtung in diverser Hinsicht als vorbildlich angesehen werden.

Die Frage der Sinnhaftigkeit der DMP steht und fällt letztlich mit dem Nachweis der Wirksamkeit. Gerade mit Blick auf die Weiterentwicklung der DMP ab 2009 unter den Rahmenbedingungen des Gesundheitsfonds und morbiditätsorientierten Risikostrukturausgleichs („MorbiRSA“) ist diese Frage in zweierlei Hinsicht von Bedeutung:

1) Können neben einer Verbesserung der medizinischen Versorgung durch die DMP ökonomische Effekte erreicht werden, die es aus einzelwirtschaftlicher Sicht einer Krankenkasse im Wettbewerb attraktiv erscheinen lassen, DMP weiterhin anzubieten?

2) Sind gesamtgesellschaftliche Effekte in der Versorgungsqualität und -wirtschaftlichkeit so deutlich, dass eine mittelbare Förderung der DMP durch die Politik gerechtfertigt bzw. sogar notwendig ist?

Die bisher publizierten Ergebnisse aus den so genannten Qualitätsberichten der jeweiligen Vertragspartner auf Länderebene sowie aus der gesetzlichen Evaluation zeigen für das DMP Diabetes mellitus Typ 2 folgenden Trend: Insgesamt ist eine Verbesserung der Blutdruckwerte bei hypertonen Patienten im Programmverlauf erkennbar, während beim Blutzucker (HbA1c) bereits zu Programmbeginn so niedrige Durchschnittswerte ermittelt wurden, dass nur ein geringes Verbesserungspotenzial eingeräumt werden $\mathrm{kann}^{8}$. Im Bereich der Prozessqualität zeigen sich zudem hohe Quoten an Patienten, die mindestens einmal pro Jahr die obligatorische Fußuntersuchung erhalten und einmal jährlich zur augenärztlichen Untersuchung überwiesen werden.

Die methodische Kritik an diesen Resultaten bezieht sich neben Fragen der Datenvalidität in erster Linie auf das Fehlen einer Vergleichsgruppe von Nicht-DMP-Teilnehmern. Alle dargestellten Ergebnisse basieren auf den im Rahmen der DMP erstellten Dokumentationsdaten, die wiederum nur bei freiwilliger Teilnahme des Arztes und des Patienten erhoben werden dürfen. Für nichtteilnehmende Ärzte und deren Patienten liegen somit zwangsläufig keine vergleichbaren Daten vor, obwohl gerade der Unterschied zwischen der Versorgung innerhalb der DMP und der „Regelversorgung ohne DMP“ die meisten Rückschlüsse über den „Wert“ der DMP erbringen würde $^{9,10}$. Dieses systemimmanente Defizit lässt sich folglich nur beheben, wenn a) auf andere Datenquellen zurückgegriffen wird oder b) eine generelle Pflicht zur Erhebung von Qualitätssicherungsdaten in der ambulanten Chronikerversorgung geschaffen würde. Während letztere eine wohl eher theoretische Option für die Zukunft darstellt, ist die Erhebung von Vergleichsdaten z.B. anhand von Patientenbefragungen oder Routinedaten der
GKV für die zurückliegenden Zeiträume seit DMP-Einführung durchaus praktikabel und aussagekräftig.

Retrospektive Datenvergleiche von teilnehmenden und nichtteilnehmenden Versicherten müssen natürlich mit Vorsicht im Hinblick auf Selektionseffekte und sonstige Einflussfaktoren, die außerhalb der eigentlichen Studienhypothesen liegen, betrachtet werden. Dabei zeigt sich, dass u.a. deshalb nicht alle Einflussfaktoren kontrollierbar sind, weil diese auch Teil der zu messenden Ergebnisparameter selbst sein können. So resumierten die Autoren einer ersten Datenauswertung der RSA-Profile eingeschriebener Versicherter im Jahr 2003, dass bei der Einschreibung von Typ-2-Diabetikern „jüngere und weniger schwer betroffene Patienten dominieren" und daher der Verdacht einer „Rosinenpickerei“ naheliege ${ }^{11}$. Die geringere Morbidität wurde dort mit einem niedrigeren Leistungsausgabenprofil gegenüber zuvor publizierten Ausgabenprofilen von Diabetikern, insbesondere im Bereich der Krankenhausleistungen begründet. Da im Jahr 2003 ein „quasi prä-interventioneller Zustand“ vorlag, wurde ein Programmeffekt bei der Ergebnisinterpretation ausgeschlossen. Mit zunehmender Programmdauer ist hingegen zu erwarten, dass sich die eigentlichen Programmeffekte und mögliche Selektionseffekte insoweit überlagern ${ }^{12}$.

Nachfolgend werden die Ergebnisse einer vergleichenden Auswertung von Leistungsdaten der BARMER Ersatzkasse dargestellt und im hier aufgezeigten Kontext diskutiert. Eine zweite, ebenfalls kürzlich bei der BARMER durchgeführte vergleichende Erhebung mittels Patientenbefragung (s. Elkeles et al. in diesem Heft) gibt weitere Aufschlüsse über die Versorgungsmerkmale von eingeschriebenen und nichteingeschriebenen Diabetikern.

\section{Material, Methodik und Ergebnisse der BARMER-Leistungsdatenanalyse}

Erstmals wurden Leistungsdaten der gesetzlichen Krankenversicherung von Patienten mit Diabetes mellitus mit und ohne Teilnahme an den DMP Diabetes mellitus Typ 1 und 2 vergleichend analysiert. Es wurde untersucht, ob sich bezüglich diabetesspezifischer Komplikationen und Begleiterkrankungen Unterschiede zwischen den beiden Gruppen bei Krankenhausbehandlung, Augenarztkontakten und Arzneimittelversorgung erkennen lassen. Grundlage bildeten Leistungsdaten aus den Jahren 2005 und 2006, die der BARMER Ersatzkasse gem. § 294, § 300 und $\S 301$ SGB V übermittelt werden. Dabei wurden Diagnoseschlüssel (ICD-10), Krankenhaus-Operationsschlüssel (OPS) und verordnete Arzneimittel (Selektion nach ATCCode) untersucht, die diabetesspezifische Versorgungsmerkmale abbilden.

Bundesweit wurden 80.745 Versicherte der BARMER, die seit 2005 ununterbrochen am DMP Diabetes teilnehmen sowie eine Vergleichsgruppe von 79.137 Diabetikern, die noch nie am DMP teilgenommen haben, in die pseudonymisierte Auswertung eingeschlossen. Ausgewählt wurden Personen im Alter zwischen 40 und 
95 Jahren, die im Jahr 2005 mindestens dreimal ein Antidiabetikum verordnet bekamen und zum Zeitpunkt der Untersuchung, Mai 2007, einen laufenden Leistungsanspruch hatten. Weitere 61.895 Patienten, die auf diese Weise ermittelt wurden und deren DMP Unterlagen unvollständig waren, deren DMP-Teilnahme unterbrochen wurde oder deren Teilnahmebeginn nach dem 01.01.2006 lag, wurden bei der vergleichenden Auswertung nicht berücksichtigt.

Um alters- und geschlechtsspezifische Einflüsse beim Vergleich von DMP-Teilnehmern versus Nichtteilnehmern auszuschließen, jedoch gleichzeitig genderbedingte Unterschiede abzubilden, wurde jeweils für Männer und Frauen eine getrennte, altersstandardisierte Auswertung vorgenommen. Da eine etablierte Standardpopulation für Diabetiker in Deutschland nicht bekannt ist, wurde jeweils eine männliche und weibliche Standardpopulation aus der Summe der definierten DMP-Teilnehmer und Nichtteilnehmer mit einer Altersklassenzuordnung in 5-JahresSchritten gebildet. Diese Populationen bildeten den Bezug für die direkte Altersstandardisierung. Die in den Ergebnissen ermittelten Erwartungswerte gelten jeweils für 1000 Personen der jeweiligen Standardpopulation.

Die Ergebnisse für beide Gruppen wurden zunächst im Sinne einer deskriptiven Statistik gegenübergestellt. Hierbei wurden altersstandardisierte Summen betrachtet (Krankenhausfallzahlen, Krankenhausverweildauertage, Krankenhauskosten). Im nächsten Schritt wurden altersstandardisierte Raten ermittelt und auf Signifikanz überprüft (durch Berechnung der 95\%-Konfidenzintervalle analog zur Berechnung der Konfidenzintervalle bei Inzidenz- oder Mortalitätsraten).

Zunächst zeigt diese Datenanalyse einen Unterschied bei der Alters- und Geschlechtsverteilung beider Gruppen, der besonders bei den älteren Versicherten imponiert, die schon alleine aufgrund des fortgeschrittenen Lebensalters ein erhöhtes Risiko für die untersuchten Komplikationen tragen. So lag das Verhältnis von DMP-Teilnehmern zu Nichtteilnehmern bei männlichen Versicherten bis 80 Jahre bei 5,2 zu 4,8 (Frauen: 5,7 zu $4,3)$. Bei den über achtzigjährigen Männern lag dieses Verhältnis bei 3,9 zu 6,1 (Frauen: 3,3 zu 6,7). Diese Unterschiede wurden wie beschrieben kontrolliert und haben somit keinen Einfluss auf weitere Ergebnisdarstellung.

Die Gegenüberstellung der Summe aller im Beobachtungszeitraum angefallenen Krankenhausaufenthalte, Krankenhausverweildauertage und Krankenhauskosten zeigt durchgängig niedrigere Werte für die DMP-Teilnehmer. Hinsichtlich typischer Folgeerkrankungen des Diabetes mellitus wurden die Krankenhausfälle mit entsprechenden Hauptentlassungsdiagnosen ausgewertet (KHK, Herzinsuffizienz als häufige Folge der KHK und Schlaganfälle). Auffällig ist hier, dass in der Gruppe der Programmteilnehmer häufiger Krankenhausaufenthalte wegen eines weniger kritischen Stadiums der KHK (Angina pectoris, Chronisch ischämische Herzkrankheit) vorkamen. Nichtteilnehmer wurden hingegen häufiger wegen fortgeschrittener Komplikationen (Myokardinfarkt, Herzinsuffizienz, Schlaganfall, Major- und Minoramputationen) im Krankenhaus behandelt (Tabelle 1).

Um die Frage nach statistisch signifikanten Unterschieden zwischen Programmteilnehmern und Nichtteilnehmern zu untersuchen, wurden für die genannten Folgeerkrankungen Raten gebildet: Ermittelt wurde die Anzahl von Personen, die im Jahr 2006 mindestens einmal wegen einer entsprechenden Diagnose stationär behandelt werden mussten. Die Berechnung des 95\%-Konfidenzintervalls erfolgte mit dem bereits benannten Verfahren (Tabelle 2).
Tabelle 1: Ermittelte Parameter aus Krankenhausfällen 2006, u.a. nach Hauptentlassungsdiagnosen und Prozeduren

\begin{tabular}{|c|c|c|c|c|}
\hline & \multicolumn{4}{|c|}{$\begin{array}{l}\text { Alle Angaben sind Erwartungswerte für } 1000 \text { Versicherte der } \\
\text { Diabetiker-Standardpopulation }\end{array}$} \\
\hline & \multicolumn{2}{|c|}{ Männer } & \multicolumn{2}{|c|}{ Frauen } \\
\hline & DMP & kein DMP & DMP & kein DMP \\
\hline Summe aller KH-Fälle & 513 & 608 & 509 & 634 \\
\hline Summe aller KHVWT & 3.674 & 4.564 & 3.787 & 5.202 \\
\hline Summe aller $\mathrm{KH}$-Kosten in $€$ & 1.429 .111 & 1.702 .086 & 1.279 .593 & 1.698 .605 \\
\hline $\begin{array}{l}\text { Summe KH- Fälle: Angina pectoris } \\
\text { (HED ICD I20) }\end{array}$ & 23,3 & 20,0 & 14,0 & 11,2 \\
\hline $\begin{array}{l}\text { Summe KH- Fälle: Instabile Angina pectoris } \\
\text { (HED ICD I20.0; Teilmenge von I20) }\end{array}$ & 11,3 & 9,8 & 6,6 & 5,7 \\
\hline $\begin{array}{l}\text { Summe KH- Fälle: Myokardinfarkt } \\
\text { (HED ICD I21-I23) }\end{array}$ & 9,2 & 11,0 & 6,9 & 7,9 \\
\hline $\begin{array}{l}\text { Summe KH- Fälle: Chron.ischäm. } \\
\text { Herzkrankheit (HED ICD I25) }\end{array}$ & 22,6 & 20,3 & 9,6 & 9,0 \\
\hline $\begin{array}{l}\text { Summe KH- Fälle: Herzinsuffizienz } \\
\text { (HED ICD I50) }\end{array}$ & 14,5 & 17,2 & 13,5 & 16,9 \\
\hline $\begin{array}{l}\text { Summe KH- Fälle: Schlaganfall } \\
\text { (HED ICD I63-I64) }\end{array}$ & 8,8 & 12,7 & 7,8 & 12,4 \\
\hline $\begin{array}{l}\text { Summe Minor- und Majoramputationen } \\
\text { (OPS 5-864 bis 5-865) }\end{array}$ & 5,6 & 9,1 & 1,8 & 4,7 \\
\hline
\end{tabular}

$\mathrm{KH}=$ Krankenhaus, $\mathrm{KHVWT}=$ Krankenhausverweildauertage, $\mathrm{HED}=$ Hauptentlassungsdiagnose Quelle: BARMER 
Es zeigen sich eindrucksvolle Unterschiede: Die Zahl der Personen, die wegen Schlaganfällen im Krankenhaus behandelt wurden, lag im Jahr 2006 bei DMP-Teilnehmern rund ein Drittel niedriger als bei Nichtteilnehmern. Bei der Zahl der von Major- oder Minoramputationen betroffenen Patienten ergibt sich sogar ein noch deutlicherer Vorteil zugunsten der DMP-Teilnehmer. Für am DMP teilnehmende Frauen zeigt sich darüber hinaus, dass signifikant weniger Personen wegen Herzinsuffizienz, hingegen aber signifikant mehr Frauen wegen Angina pectoris im Krankenhaus waren.

Bei der Auswertung ambulanter Behandlungsdaten (KV-Abrechnungsdaten) zeigte sich zudem, dass die Zahl der für eine leitliniengerechte Vorsorge notwendigen augenärztlichen Kontakte bei DMP-Teilnehmern signifikant höher lag. Von 1000 Diabetikern hatten 780 Teilnehmer $\left(\mathrm{KI}_{95 \%}=777-783\right)$ und nur $538\left(\mathrm{KI}_{95 \%}=535-542\right)$ Nichtteilnehmer mindestens einen Augenarztkontakt im Jahr 2006.

Schließlich zeigt auch die Auswertung von Apothekenabrechnungsdaten einen signifikanten Unterschied zwischen DMP-Teilnehmern und Nichtteilnehmern. Es wurde untersucht, welcher Anteil der Versicherten aus den beiden Gruppen in den Jahren 2005 und 2006 mindestens einmal eine Medikation erhielt, die gemäß Behandlungsleitlinien als protektiv hinsichtlich makrovaskulärer Komplikationen einzustufen ist. Für beide Jahre liegt diese Zahl, mit Ausnahme der Thrombozytenaggregationshemmer bei Frauen, bei den DMP-Teilnehmern höher (Tabelle 3).

Tabelle 2: Anzahl Personen mit mindestens einem Krankenhausaufenthalt in 2006 wegen

\begin{tabular}{|c|c|c|c|c|}
\hline & \multicolumn{4}{|c|}{$\begin{array}{c}\text { Alle Angaben sind Erwartungswerte für } 1000 \text { Versicherte } \\
\text { der Diabetiker-Standardpopulation } \\
(95 \% \text { Konfidenzntervall = Wert +/- Klammerbetrag) }\end{array}$} \\
\hline & \multicolumn{2}{|c|}{ Männer } & \multicolumn{2}{|c|}{ Frauen } \\
\hline & DMP & kein DMP & DMP & kein DMP \\
\hline $\begin{array}{l}\text { Angina pectoris } \\
\text { (HED ICD I20) }\end{array}$ & $\begin{array}{r}19,66 \\
(+/-1,42)\end{array}$ & $\begin{array}{r}17,36 \\
(+/-1,83)\end{array}$ & $\begin{array}{r}12,25 \\
(+/-1,07)\end{array}$ & $\begin{array}{r}10,01 \\
(+/-0,98)\end{array}$ \\
\hline $\begin{array}{l}\text { Instabile Angina pectoris } \\
\text { (HED ICD I20.0; Teilmenge von I20) }\end{array}$ & $\begin{array}{r}9,76 \\
(+/-1,01)\end{array}$ & $\begin{array}{r}8,97 \\
(+/-0,99)\end{array}$ & $\begin{array}{r}5,93 \\
(+/-0,76)\end{array}$ & $\begin{array}{r}5,21 \\
(+/-0,71)\end{array}$ \\
\hline $\begin{array}{l}\text { Myokardinfarkt } \\
\text { (HED ICD I21-I23) }\end{array}$ & $\begin{array}{r}7,50 \\
(+/-0,90)\end{array}$ & $\begin{array}{r}8,52 \\
(+/-0,96)\end{array}$ & $\begin{array}{r}5,55 \\
(+/-0,76)\end{array}$ & $\begin{array}{r}6,12 \\
(+/-0,74)\end{array}$ \\
\hline $\begin{array}{l}\text { Chronisch ischämische Herzkrankheit } \\
\text { (HED ICD I25) }\end{array}$ & $\begin{array}{r}18,58 \\
(+/-1,36)\end{array}$ & $\begin{array}{r}16,62 \\
(+/-1,36)\end{array}$ & $\begin{array}{r}8,20 \\
(+/-0,86)\end{array}$ & $\begin{array}{r}7,46 \\
(+/-0,86)\end{array}$ \\
\hline $\begin{array}{l}\text { Herzinsuffizienz } \\
\text { (HED ICD I50) }\end{array}$ & $\begin{array}{r}12,39 \\
(+/-1,15)\end{array}$ & $\begin{array}{r}14,22 \\
(+/-1,22)\end{array}$ & $\begin{array}{r}11,68 \\
(+/-1,19)\end{array}$ & $\begin{array}{r}14,43 \\
(+/-1,09)\end{array}$ \\
\hline $\begin{array}{l}\text { Hirninfarkt, Schlaganfall* } \\
\text { (HED ICD I63-I64) }\end{array}$ & $\begin{array}{r}8,12 \\
(+/-0,94)\end{array}$ & $\begin{array}{r}11,41 \\
(+/-1,10)\end{array}$ & $\begin{array}{r}7,15 \\
(+/-0,91)\end{array}$ & $\begin{array}{r}11,12 \\
(+/-0,97)\end{array}$ \\
\hline $\begin{array}{l}\text { Minor- und Majoramputationen* } \\
\text { (OPS 5-864 bis 5-865) }\end{array}$ & $\begin{array}{r}4,50 \\
(+/-0,71)\end{array}$ & $\begin{array}{r}7,26 \\
(+/-0,90)\end{array}$ & $\begin{array}{r}1,61 \\
(+/-0,41)\end{array}$ & $\begin{array}{r}3,84 \\
(+/-0,58)\end{array}$ \\
\hline
\end{tabular}

$\mathrm{HED}=$ Hauptentlassungsdiagnose

* signifikanter Unterschied DMP-Teilnehmer vs. Nichtteilnehmer

** signifikanter Unterschied DMP-Teilnehmer vs. Nichtteilnehmer bei Frauen

Quelle: BARMER

\section{Diskussion der Ergebnisse der BARMER-Leistungsdatenanalyse}

Die Ergebnisse zur Arzneimittelversorgung sind besonders bedeutsam im Hinblick auf die Interpretation der zuvor dargestellten niedrigeren Raten an Krankenhausbehandlung wegen Schlaganfall und Amputationen. Die geringeren Komplikationsraten bei DMP-Teilnehmern weisen auf den ersten Blick auf eine insgesamt niedrigere Morbidität dieser Gruppe hin. Da dies zugleich der Zielsetzung der Programme entspricht, gilt es zwischen einer a priori Selektion „gesünderer" Patienten und programminduzierten Effekten zu unterschieden.

Die Bewertung von Morbidität wird nach den international verfügbaren Klassifikationssystemen, die auch für den „Morbi-RSA“ ab 2009 maßgebend sind, vorrangig anhand von ambulanten und stationären Diagnosen sowie Medikationsdaten vorgenommen ${ }^{13}$. Da in der hier dargestellten Auswertung DMP-Teilnehmer nahezu durchgängig häufiger spezifische Medikamente erhielten, könnte dies somit Ausdruck einer insgesamt höheren Morbidität der DMP-Teilnehmer, bezogen auf die hier relevanten Begleit- und Folgeerkrankungen sein. Umso eindrucksvoller wären dann die geringeren Ereignisraten bei Schlaganfällen und Amputationen im Krankenhaus. Das würde also bedeuten, dass die Programmteilnehmer trotz höherer Morbidität seltener schwerwiegende Komplikationen mit Krankenhausbehandlung haben.

Die zweite mögliche Schlussfolgerung ist, dass DMPTeilnehmer nicht aufgrund einer höheren Prävalenz der arteriellen Hypertonie, Dyslipidämie bzw. KHK häufiger mit den entsprechenden Medikamenten behandelt werden. In diesem Fall müsste von einer morbiditätsunabhängigen Varianz in der Versorgung zwischen beiden Gruppen ausgegangen werden, die den Schluss einer Unterversorgung mit protektiven Medikamenten in der Gruppe der NichtDMP-Patienten nahelegt. Damit wären allerdings DMP-Patienten bezogen auf die Arzneimittelversorgung „besser versorgt".

Für einen qualitativen Unterschied 
Tabelle 3: Anzahl Personen, die im Jahr 2006, bzw. 2005 mindestens einmal eine Verordnung für ein aufgeführtes Medikament erhielten

\begin{tabular}{|c|c|c|c|c|}
\hline & \multicolumn{4}{|c|}{$\begin{array}{l}\text { Alle Angaben sind Erwartungswerte für } 1000 \text { Versicherte der Diabetiker- } \\
\text { Standardpopulation ( } 95 \% \text { Konfidenzintervall }=\text { Wert }+/- \text { Klammerbetrag) }\end{array}$} \\
\hline & \multicolumn{2}{|c|}{ Männer } & \multicolumn{2}{|c|}{ Frauen } \\
\hline & DMP & kein DMP & DMP & kein DMP \\
\hline $\begin{array}{l}\text { 2006: Leitliniengerechte Wirkstoffgruppen zur Hypertoniebehandlung } \\
\text { (Diuretika, ACE-Hemmer, } \beta 1 \text {-selektive Betablocker) }\end{array}$ & $\begin{array}{l}759,47 \\
(+/-4,40)\end{array}$ & $\begin{array}{l}717,60 \\
(+/-4,57)\end{array}$ & $\begin{array}{l}781,83 \\
(+/-3,92)\end{array}$ & $\begin{array}{l}755,18 \\
(+/-4,09)\end{array}$ \\
\hline $\begin{array}{l}\text { 2006: explizit in Leitlinien benannte Wirkstoffe zur Hypertonie- } \\
\text { behandlung (z.B. Hydrochlorothiazid, Metoprolol, Captopril) }\end{array}$ & $\begin{array}{c}652,88 \\
(+/-4,93)\end{array}$ & $\begin{array}{c}614,62 \\
(+/-5,00)\end{array}$ & $\begin{array}{l}669,05 \\
(+/-4,57)\end{array}$ & $\begin{array}{c}635,15 \\
(+/-4,60)\end{array}$ \\
\hline 2006: Thrombozytenaggregationshemmer & $\begin{array}{c}221,38 \\
(+/-4,23)\end{array}$ & $\begin{array}{c}212,25 \\
(+/-4,23)\end{array}$ & $\begin{array}{c}151,69 \\
(+/-3,61)\end{array}$ & $\begin{array}{c}157,04 \\
(+/-3,40)\end{array}$ \\
\hline 2006: Koronardilatatoren (z.B. Nitrate, Molsidomin) & $\begin{array}{c}145,31 \\
(+/-3,58)\end{array}$ & $\begin{array}{c}133,58 \\
(+/-3,48)\end{array}$ & $\begin{array}{c}151,58 \\
(+/-3,62)\end{array}$ & $\begin{array}{c}134,43 \\
(+/-3,14)\end{array}$ \\
\hline 2006: Statine & $\begin{array}{c}426,67 \\
(+/-5,08)\end{array}$ & $\begin{array}{c}352,64 \\
(+/-4,97)\end{array}$ & $\begin{array}{c}382,57 \\
(+/-4,64)\end{array}$ & $\begin{array}{c}296,41 \\
(+/-4,43)\end{array}$ \\
\hline $\begin{array}{l}\text { 2006: explizit in Leitlinien benannte Statine (Pravastatin, Simvastatin, } \\
\text { Atorvastatin) }\end{array}$ & $\begin{array}{c}387,07 \\
(+/-5,00) \\
\end{array}$ & $\begin{array}{c}317,62 \\
(+/-4,85) \\
\end{array}$ & $\begin{array}{c}349,65 \\
(+/-4,56) \\
\end{array}$ & $\begin{array}{c}267,99 \\
(+/-4,31)\end{array}$ \\
\hline $\begin{array}{l}\text { 2005: Leitliniengerechte Wirkstoffgruppen zur Hypertoniebehandlung } \\
\text { (Diuretika, ACE-Hemmer, } \beta 1 \text {-selektive Betablocker) }\end{array}$ & $\begin{array}{l}736,00 \\
(+/-4,52)\end{array}$ & $\begin{array}{c}695,31 \\
(+/-4,67)\end{array}$ & $\begin{array}{l}765,34 \\
(+/-4,03)\end{array}$ & $\begin{array}{l}742,30 \\
(+/-4,16)\end{array}$ \\
\hline $\begin{array}{l}\text { 2005: explizit in Leitlinien benannte Wirkstoffe zur Hypertonie- } \\
\text { behandlung (z.B. Hydrochlorothiazid, Metoprolol, Captopril) }\end{array}$ & $\begin{array}{c}629,23 \\
(+/-4,98)\end{array}$ & $\begin{array}{l}593,39 \\
(+/-5,05)\end{array}$ & $\begin{array}{l}651,46 \\
(+/-4,63)\end{array}$ & $\begin{array}{l}621,82 \\
(+/-4,64)\end{array}$ \\
\hline 2005: Thrombozytenaggregationshemmer & $\begin{array}{c}206,99 \\
(+/-4,13)\end{array}$ & $\begin{array}{c}197,76 \\
(+/-4,13)\end{array}$ & $\begin{array}{c}141,62 \\
(+/-3,51)\end{array}$ & $\begin{array}{c}147,17 \\
(+/-3,31)\end{array}$ \\
\hline 2005: Koronardilatatoren (z.B. Nitrate, Molsidomin) & $\begin{array}{c}149,95 \\
(+/-3,62)\end{array}$ & $\begin{array}{c}138,64 \\
(+/-3,53)\end{array}$ & $\begin{array}{c}154,57 \\
(+/-3,64)\end{array}$ & $\begin{array}{c}139,28 \\
(+/-3,19)\end{array}$ \\
\hline 2005: Statine & $\begin{array}{c}378,81 \\
(+/-4,98)\end{array}$ & $\begin{array}{c}315,90 \\
(+/-4,84)\end{array}$ & $\begin{array}{c}344,96 \\
(+/-4,55)\end{array}$ & $\begin{array}{c}270,26 \\
(+/-4,31)\end{array}$ \\
\hline $\begin{array}{l}\text { 2005: explizit in Leitlinien benannte Statine (Pravastatin, Simvastatin, } \\
\text { Atorvastatin) }\end{array}$ & $\begin{array}{c}336,54 \\
(+/-4,84)\end{array}$ & $\begin{array}{c}279,41 \\
(+/-4,68)\end{array}$ & $\begin{array}{c}308,45 \\
(+/-4,43)\end{array}$ & $\begin{array}{c}240,37 \\
(+/-4,16)\end{array}$ \\
\hline
\end{tabular}

Quelle: BARMER

in der Versorgung sprechen letztlich auch die höhere Zahl an augenärztlichen Untersuchungen. Auch im Bereich der regelmäßigen Fußuntersuchungen wurde bereits im Rahmen von Patientenbefragungen festgestellt, dass ein deutlicher Unterschied zugunsten von DMP-Teilnehmern besteht ${ }^{14}$.

Zusammenfassend können somit reale Qualitätsunterschiede in der Versorgung festgestellt werden, die sich nicht ohne weiteres durch eine Risikoselektion jüngerer und „gesünderer“ Diabetiker erklären lassen. Vielmehr werden Fragen aufgeworfen, welche systematischen Unterschiede in der Versorgungssituation - bei den behandelten Patienten und nicht zuletzt bei den behandelnden Ärzten - ggf. zu diesen deutlichen Ergebnissen geführt haben.

\section{Ergänzende Informationen über Merkmale der Versorgung bei DMP-Teilnehmern und Nichtteilnehmern anhand einer vergleichenden Patientenbefragung}

Weitere Erkenntnisse im zuvor dargestellten Kontext der Untersuchung systematischer Unterschiede der Versorgung bei DMP-Teilnehmern und nichtteilnehmenden Diabetikern liefert eine aktuelle BARMER-Versichertenbefragung, mit einer Nettostichprobe von 4.340 Versicherten mit Diabetes mellitus Typ 2.
Ausgewählt wurden Versicherte im Alter von 45 bis 79 Jahren aufgrund von Verordnungsdaten bzgl. diabetesspezifischer Medikation. Eine ausführliche Darstellung der Stichprobenauswahl, der Patientenbefragung und ihrer Ergebnisse findet sich im Beitrag von Elkeles et al. in diesem Heft ${ }^{2}$.

An dieser Stelle werden nur ausgewählte Ergebnisse dargestellt, die zur Beantwortung der bislang aufgeworfenen Fragen besonders relevant erscheinen. Hierbei geht es im Kern um Selektionseffekte bzgl. der Risikostruktur und Morbidität der Patienten sowie weitere Parameter, die eine plausible Erklärung für die aufgezeigten Versorgungsunterschiede geben könnten. Dazu zählen:

- Morbidität und Therapieform: Dauer und Schwere der Diabeteserkrankung, Begleiterkrankungen, Anteil Insulinbehandlung sowie sonstige medikamentöse und nichtmedikamentöse Therapie

- Sozialstatus: Schulbildung und Ausbildung

- Informationsstand: Kenntnis des HbA1c-Wertes

- Versorgungsstruktur: Haus- und fachärztliche Behandlung, Krankenhausbehandlung

Die Auswertung der Befragungsergebnisse umfasst 2182 Nichtteilnehmer und 2158 DMP-Teilnehmer, wobei 95,6\% der Teilnehmer (2061) seit einem Jahr oder länger im DMP eingeschrieben waren. Die Angaben zur Teilnah- 
Tabelle 4: Ausgewählte Ergebnisse der Versichertenbefragung zur Versorgung bei Dianetes mellitus Typ 2

\begin{tabular}{|c|c|c|c|c|c|c|c|c|c|}
\hline \multirow{3}{*}{$\begin{aligned} & \text { TN }= \text { DMP-Teilnehmer } \\
& \text { NTN = Nichtteilnehmer } \\
& \text { T-Wert: Signifikante E rgebnisse sind mit } \\
& \quad \text { Sternchen }\left(^{*}\right) \text { gekennzeichnet }\end{aligned}$} & \multicolumn{6}{|c|}{ Standardisiert nach Alter } & \multirow{2}{*}{\multicolumn{3}{|c|}{$\begin{array}{c}\text { Standardisiert nach } \\
\text { Alter und Geschlecht } \\
\text { Männer \& Frauen }\end{array}$}} \\
\hline & \multicolumn{3}{|c|}{ Männer } & \multicolumn{3}{|c|}{ Frauen } & & & \\
\hline & $\begin{array}{c}1185 \\
\text { TN }\end{array}$ & $\begin{array}{l}1181 \\
\text { NTN }\end{array}$ & T-Wert & $\begin{array}{l}973 \\
\text { TN }\end{array}$ & $\begin{array}{l}1001 \\
\text { NTN }\end{array}$ & T-Wert & $\begin{array}{l}2158 \\
\text { TN }\end{array}$ & $\begin{array}{l}2182 \\
\text { NTN }\end{array}$ & T-Wert \\
\hline \multicolumn{10}{|c|}{ Welche der folgenden Krankheiten bzw. Beschwerden hatten Sie in den letzten 12 Monaten? } \\
\hline E rhöhter Blutdruck & $66,5 \%$ & $59,1 \%$ & $3,71^{*}$ & $69,1 \%$ & $63,2 \%$ & $2,78 *$ & $67,8 \%$ & $61,2 \%$ & $4,57 *$ \\
\hline Erhöhte Blutfettwerte & $34,4 \%$ & $30,7 \%$ & 1,90 & $40,6 \%$ & $38,6 \%$ & 0,91 & $37,6 \%$ & $34,8 \%$ & 1,92 \\
\hline $\begin{array}{l}\text { Druckstellen / Geschwüre an den Füßen } \\
\text { (Diabetisches Fußsyndrom) }\end{array}$ & $8,3 \%$ & $9,9 \%$ & 1,43 & $8,7 \%$ & $7,5 \%$ & 0,99 & $8,5 \%$ & $8,7 \%$ & 0,22 \\
\hline Verengung der Herzkranzgefäße / Angina pectoris & $13,7 \%$ & $10,7 \%$ & $2,25 *$ & $10,0 \%$ & $9,9 \%$ & 0,14 & $11,8 \%$ & $10,3 \%$ & 1,64 \\
\hline Herzinfarkt & $3,0 \%$ & $3,0 \%$ & 0,01 & $1,2 \%$ & $1,6 \%$ & 0,84 & $2,1 \%$ & $2,3 \%$ & 0,52 \\
\hline Schlaganfall & $2,1 \%$ & $3,0 \%$ & 1,26 & $1,6 \%$ & $2,4 \%$ & 1,38 & $1,8 \%$ & $2,7 \%$ & 1,87 \\
\hline Nierenschaden (Nephropathie) & $6,0 \%$ & $7,3 \%$ & 1,22 & $4,8 \%$ & $5,5 \%$ & 0,74 & $5,4 \%$ & $6,4 \%$ & 1,38 \\
\hline Augenschaden (Retinopathie) & $15,0 \%$ & $15,8 \%$ & 0,53 & $15,1 \%$ & $16,3 \%$ & 0,73 & $15,0 \%$ & $16,0 \%$ & 0,91 \\
\hline Neuropathie & $21,9 \%$ & $19,9 \%$ & 1,22 & $19,2 \%$ & $17,0 \%$ & 1,27 & $20,5 \%$ & $18,4 \%$ & 1,76 \\
\hline Sonstige Erkrankungen des Herzkreislaufsystems & $14,1 \%$ & $9,9 \%$ & $3,15 *$ & $11,8 \%$ & $8,6 \%$ & $2,31 *$ & $12,9 \%$ & $9,2 \%$ & $3,84 *$ \\
\hline $\begin{array}{l}\text { Asthma bronchiale (Bronchialasthma, } \\
\text { Lungenasthma, allergisches Asthma) }\end{array}$ & $5,0 \%$ & $5,4 \%$ & 0,48 & $7,1 \%$ & $7,1 \%$ & 0,01 & $6,1 \%$ & $6,3 \%$ & 0,28 \\
\hline $\begin{array}{l}\text { Chronische Bronchitis (Husten nachts ohne } \\
\text { Erkältung und mit morgendlichem Auswurf) }\end{array}$ & $10,7 \%$ & $9,7 \%$ & 0,82 & $9,4 \%$ & $10,3 \%$ & 0,69 & $10,0 \%$ & $10,0 \%$ & 0,02 \\
\hline $\begin{array}{l}\text { Erkrankungen der Gelenke, des Rückens oder der } \\
\text { Bandscheibe }\end{array}$ & $50,0 \%$ & $46,4 \%$ & 1,74 & $66,5 \%$ & $56,2 \%$ & $4,72 *$ & $58,5 \%$ & $51,5 \%$ & $4,68 *$ \\
\hline $\begin{array}{l}\text { Erkrankungen des Magens, des Darms, der Leber } \\
\text { oder sonstiger Organe }\end{array}$ & $13,8 \%$ & $11,0 \%$ & $2,05 *$ & $16,2 \%$ & $17,0 \%$ & 0,48 & $15,1 \%$ & $14,1 \%$ & 0,87 \\
\hline Allergien / Hautkrankheiten & $16,7 \%$ & $12,5 \%$ & $2,86 *$ & $20,5 \%$ & $15,1 \%$ & $3,18 *$ & $18,7 \%$ & $13,8 \%$ & $4,32 *$ \\
\hline E rkrankungen (durch) Unfälle, Verletzungen & $6,3 \%$ & $5,3 \%$ & 1 , & $7,5 \%$ & $7,5 \%$ & 0 , & $6,9 \%$ & $6,4 \%$ & 0,65 \\
\hline Psychische Beschwerden bzw. Krankheiten & $6,8 \%$ & $6,5 \%$ & 0, & $10,0 \%$ & $10,2 \%$ & 0 & $8,5 \%$ & $8,4 \%$ & 0,03 \\
\hline \multicolumn{10}{|l|}{ Welchen Schulabschluss haben Sie? } \\
\hline Volks- oder Hauptschulabschluss & $42,1 \%$ & 43, & 0,57 & $50,0 \%$ & & 3 & $46,1 \%$ & $50,7 \%$ & $3,00 *$ \\
\hline lochschulreife & $53,9 \%$ & $51,5 \%$ & 1,19 & $44,3 \%$ & $36,2 \%$ & & $48,9 \%$ & $43,6 \%$ & $3,53 *$ \\
\hline chulabschluss & $3,4 \%$ & $4,6 \%$ & 1,42 & $4,8 \%$ & $4,3 \%$ & 0 , & $4,2 \%$ & $4,4 \%$ & 0,45 \\
\hline Keine Angabe & $0,6 \%$ & $0,7 \%$ & 0,42 & $0,9 \%$ & $1,8 \%$ & 1 & $0,8 \%$ & $1,3 \%$ & 1,69 \\
\hline \multicolumn{10}{|l|}{ Welche Berufs- oder Hochschulausbildung haben Sie? } \\
\hline Lehre & $28,1 \%$ & $32,5 \%$ & $2,29 *$ & $43,1 \%$ & $9 \%$ & 0,55 & $35,9 \%$ & $37,3 \%$ & 0,99 \\
\hline (Ber & $14,2 \%$ & 14, & & & & & $19,0 \%$ & $0 \%$ & 33 \\
\hline , Technikerschule) & $17,7 \%$ & $18,4 \%$ & & & & & $12,5 \%$ & $12,6 \%$ & 2 \\
\hline Bea & $2,0 \%$ & $1,7 \%$ & & & & & $1,2 \%$ & $1,1 \%$ & 7 \\
\hline nieurschule & $16,7 \%$ & $12,4 \%$ & & & & & $11,9 \%$ & $7,8 \%$ & $4,61 *$ \\
\hline imthochschule & $15,3 \%$ & $13,5 \%$ & & $4,5 \%$ & $3,5 \%$ & & $9,7 \%$ & $8,3 \%$ & 1,60 \\
\hline Keir & $1,4 \%$ & $1,9 \%$ & & $5,6 \%$ & $10,5 \%$ & & $3,6 \%$ & $6,3 \%$ & $4,23 *$ \\
\hline Andere Ausbildung & $4,0 \%$ & $4,8 \%$ & & $6,7 \%$ & $7,9 \%$ & & $5,4 \%$ & $6,4 \%$ & 1,32 \\
\hline Keine_Angabe & $0,6 \%$ & $0,9 \%$ & 0,91 & $1,0 \%$ & $3,3 \%$ & & $0,8 \%$ & $2,1 \%$ & $3,58 *$ \\
\hline \multicolumn{10}{|c|}{ Wie lange sind Sie bereits an Diabetes mellitus Typ 2 erkrankt? } \\
\hline Seit bis zu $4 \mathrm{~J}$ ahren & $13,6 \%$ & $20,5 \%$ & 4,5 & $\%$ & & 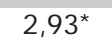 & $5,9 \%$ & $22,0 \%$ & $5,17 *$ \\
\hline Seit 5 & $37,4 \%$ & $41,2 \%$ & 1, & $34,7 \%$ & $38,8 \%$ & & $36,0 \%$ & $40,0 \%$ & $2,69 *$ \\
\hline Seit & $32,5 \%$ & $25,1 \%$ & $3,98^{*}$ & $32,3 \%$ & $26,0 \%$ & 3, & $32,4 \%$ & $25,6 \%$ & $4,97 *$ \\
\hline als $20 \mathrm{~J}$ ahren & $15,7 \%$ & $12,2 \%$ & $2,52 *$ & $13,7 \%$ & $10,5 \%$ & $2,21 *$ & $14,7 \%$ & $11,3 \%$ & $3,34 *$ \\
\hline Keine Angabe & $0,8 \%$ & $1,0 \%$ & 0 , & $1,1 \%$ & $1,2 \%$ & 0,17 & $0,9 \%$ & $1,1 \%$ & 0,47 \\
\hline \multicolumn{10}{|l|}{ Wie schwer ist Ihr Diabetes? } \\
\hline wer bis nicht schwer & $28,6 \%$ & 34, & 2, & & & $2,86^{*}$ & $8,5 \%$ & & $4,11 *$ \\
\hline & $55,0 \%$ & 51, & & & & & $\%$ & $5 \%$ & 0* \\
\hline hwer & $14,8 \%$ & $12,4 \%$ & 1, & $10,3 \%$ & $10,0 \%$ & 0,22 & $12,5 \%$ & $11,2 \%$ & 1,36 \\
\hline Keine Angabe & $1,6 \%$ & $1,8 \%$ & & & & & $2,0 \%$ & $2,0 \%$ & 8 \\
\hline \multicolumn{10}{|l|}{ W ie wird Ihr Diabetes behandelt? } \\
\hline Diät & $31,9 \%$ & & & & & & $5,2 \%$ & $30,8 \%$ & $3,08 *$ \\
\hline Tabletten & $64,4 \%$ & $70,6 \%$ & & $69,6 \%$ & $73,4 \%$ & 1, & $67,1 \%$ & $72,0 \%$ & $3,53 *$ \\
\hline Insulin & $51,4 \%$ & $42,5 \%$ & $4,37 *$ & $49,3 \%$ & $39,8 \%$ & $4,29 *$ & $50,3 \%$ & $41,1 \%$ & $6,15^{*}$ \\
\hline Bewegung & $48,3 \%$ & $38,9 \%$ & $4,62 *$ & $44,9 \%$ & $35,1 \%$ & $4,45 *$ & $46,5 \%$ & $36,9 \%$ & $6,43 *$ \\
\hline Medikation+Diät & $31,6 \%$ & $26,4 \%$ & $2,79 *$ & $37,8 \%$ & $33,7 \%$ & 1,94 & $34,8 \%$ & $30,1 \%$ & $3,29 *$ \\
\hline Medikation + Bewegung & $48,0 \%$ & $38,2 \%$ & $4,83^{*}$ & $44,1 \%$ & $34,2 \%$ & $4,54 *$ & $46,0 \%$ & $36,1 \%$ & $6,63 *$ \\
\hline Medikation + Bewegung + Diät & $22,8 \%$ & $18,3 \%$ & $2,73^{*}$ & $24,7 \%$ & $18,8 \%$ & $3,19 *$ & $23,8 \%$ & $18,5 \%$ & $4,23 *$ \\
\hline nur Tabletten & $47,7 \%$ & $56,4 \%$ & $4,21 *$ & $49,4 \%$ & $58,7 \%$ & $4,16 *$ & $48,6 \%$ & $57,5 \%$ & $5,94 *$ \\
\hline nur Insulin & $34,8 \%$ & $28,2 \%$ & $3,42 *$ & $29,0 \%$ & $25,0 \%$ & $2,00 *$ & $31,8 \%$ & $26,6 \%$ & $3,78^{*}$ \\
\hline Insulin und Ta & $16,7 \%$ & $14,2 \%$ & 1,64 & $20,3 \%$ & $14,7 \%$ & $3,25 *$ & $18,5 \%$ & $14,5 \%$ & $3,59 *$ \\
\hline Ohne Medikamente & $0,8 \%$ & $1,2 \%$ & 0,78 & $1,3 \%$ & $1,6 \%$ & 0,47 & $1,1 \%$ & $1,4 \%$ & 0,85 \\
\hline
\end{tabular}


Tabelle 4: Ausgewählte Ergebnisse der Versichertenbefragung zur Versorgung bei Dianetes mellitus Typ 2 (Forts.)

\begin{tabular}{|c|c|c|c|c|c|c|c|c|c|}
\hline \multirow{3}{*}{$\begin{aligned} \text { TN = } & \text { DMP-Teilnehmer } \\
\text { NTN = } & \text { Nichtteilnehmer } \\
\text { T-Wert: Signifikante Ergebnisse sind mit } & \text { Sternchen }\left(^{*}\right) \text { gekennzeichnet }\end{aligned}$} & \multicolumn{6}{|c|}{ Standardisiert nach Alter } & \multirow{2}{*}{\multicolumn{3}{|c|}{$\begin{array}{l}\text { Standardisiert nach } \\
\text { Alter und Geschlecht } \\
\text { Männer \& Frauen }\end{array}$}} \\
\hline & \multicolumn{3}{|c|}{ Männer } & \multicolumn{3}{|c|}{ Frauen } & & & \\
\hline & $\begin{array}{c}1185 \\
\text { TN }\end{array}$ & $\begin{array}{l}1181 \\
\text { NTN }\end{array}$ & T-Wert & $\begin{array}{l}973 \\
\text { TN }\end{array}$ & $\begin{array}{l}1001 \\
\text { NTN }\end{array}$ & T-Wert & $\begin{array}{c}2158 \\
\text { TN }\end{array}$ & $\begin{array}{l}2182 \\
\text { NTN }\end{array}$ & T-Wert \\
\hline \multicolumn{10}{|c|}{ Wo waren Sie in den letzten 12 Monaten wegen Ihres Diabetes in Behandlung? } \\
\hline $\begin{array}{l}\text { Allgemeinarzt / praktischer Arzt } \\
\text { Internist (kein Diabetologe) }\end{array}$ & $\begin{array}{l}63,2 \% \\
22,5 \%\end{array}$ & $\begin{array}{l}65,0 \% \\
22,0 \%\end{array}$ & $\begin{array}{l}0,92 \\
0,28\end{array}$ & $\begin{array}{l}59,0 \% \\
21,5 \%\end{array}$ & $\begin{array}{l}63,3 \% \\
24,1 \%\end{array}$ & $\begin{array}{l}1,97 * \\
1,36\end{array}$ & $\begin{array}{l}61,0 \% \\
22,0 \%\end{array}$ & $\begin{array}{l}64,1 \% \\
23,1 \%\end{array}$ & $\begin{array}{l}2,12^{*} \\
0,87\end{array}$ \\
\hline $\begin{array}{l}\text { Allgemeinarzt / praktischer Arzt oder Internist (kein } \\
\text { Diabetologe) }\end{array}$ & $78,7 \%$ & $82,5 \%$ & $2,34 *$ & $75,6 \%$ & $83,4 \%$ & $4,32 *$ & $77,1 \%$ & $83,0 \%$ & $4,85^{*}$ \\
\hline Diabetologische S chwerpunktpraxis / Diabetologe & $39,2 \%$ & $21,4 \%$ & $9,56 *$ & $40,3 \%$ & $20,1 \%$ & $9,99 *$ & $39,8 \%$ & $20,8 \%$ & $13,91^{*}$ \\
\hline $\begin{array}{l}\text { Nierenspezialist (Nephrologe) } \\
\text { Augenarzt } \\
\text { Krankenhaus }\end{array}$ & $\begin{array}{c}9,0 \% \\
77,3 \% \\
8,7 \%\end{array}$ & $\begin{array}{l}8,9 \% \\
59,3 \% \\
11,9 \%\end{array}$ & $\begin{array}{l}0,11 \\
9,59 * \\
2,56^{*}\end{array}$ & $\begin{array}{c}8,1 \% \\
78,9 \% \\
8,0 \%\end{array}$ & $\begin{array}{c}7,0 \% \\
60,4 \% \\
8,7 \%\end{array}$ & $\begin{array}{l}0,98 \\
9,14^{*} \\
0,50\end{array}$ & $\begin{array}{c}8,6 \% \\
78,1 \% \\
8,3 \%\end{array}$ & $\begin{array}{r}7,9 \% \\
59,9 \% \\
10,2 \%\end{array}$ & $\begin{array}{c}0,79 \\
13,28^{*} \\
2,12^{*}\end{array}$ \\
\hline \multicolumn{10}{|l|}{ Kennen Sie Ihren HbAlc-Wert? } \\
\hline $\begin{array}{l}\text { HbAlc bekannt } \\
\text { HbAlc nicht bekannt } \\
\text { Keine Angabe }\end{array}$ & $\begin{array}{c}80,6 \% \\
14,9 \% \\
4,5 \%\end{array}$ & $\begin{array}{c}56,0 \% \\
37,4 \% \\
6,6 \%\end{array}$ & $\begin{array}{c}13,37 * \\
12,89^{*} \\
2,28 *\end{array}$ & $\begin{array}{c}79,1 \% \\
16,1 \% \\
4,8 \%\end{array}$ & $\begin{array}{c}55,6 \% \\
35,7 \% \\
8,6 \%\end{array}$ & $\begin{array}{c}11,47^{*} \\
10,21^{*} \\
3,40^{*}\end{array}$ & $\begin{array}{c}79,8 \% \\
15,5 \% \\
4,7 \%\end{array}$ & $\begin{array}{c}55,8 \% \\
36,5 \% \\
7,7 \%\end{array}$ & $\begin{array}{c}17,53^{*} \\
16,26^{*} \\
4,13^{*}\end{array}$ \\
\hline
\end{tabular}

medauer blieben bei den nachfolgenden Auswertungen jedoch unberücksichtigt.

Um alters- und geschlechtsspezifische Einflussfaktoren zu kontrollieren, wurden die nachfolgend dargestellten Resultate alters- und geschlechtsstandardisiert, bzw. nach Geschlechtern differenziert ermittelt. Die Standardpopulation wurde hierbei aus 97.864 DMP-Teilnehmern mit diabetesspezifischen Arzneimittelverordnungen gebildet. Dabei erfolgte eine Altersklassenzuordnung zu den Gruppen 45-59, 60-69 und 70-79 Jahre.

Zur Überprüfung der statistischen Bedeutung von Differenzen zwischen Teilnehmern und Nichtteilnehmern wurde der T-Test für Anteilswerte gerechnet, wobei von einem Sicherheitsgrad von 95\% bzw. einer Irrtumswahrscheinlichkeit von 5\% ( $=0,05)$ ausgegangen wird. Bei einem angegebenen T-Wert $>1,96$ wird von einem signifikanten Unterschied zwischen Teilnehmern und Nichtteilnehmern ausgegangen. Die statistische Aufbereitung der Befragungsergebnisse erfolgte nach Vorgaben der FB+E Forschung, Beratung + Evaluation GmbH Berlin.

Die ausgewählten Ergebnisse können der Tabelle 4 entnommen werden.

\subsection{Morbidität und Therapieform}

Aus den bereits dargestellten Ergebnissen der Leistungsdatenauswertung ergab sich u.a. die Frage, ob die häufigere Verordnung spezifischer Medikamente bei DMP-Teilnehmern Ausdruck einer insgesamt höheren Morbidität mit einem höheren Risiko für Komplikationen bei diesen Versicherten zu werten ist. In diesem Fall wären die geringeren Ereignisraten bei z.B. Schlaganfällen in dieser Gruppe um so eindrucksvoller.

Die Frage nach der Dauer der Diabetes- erkrankung beantworteten 15,9\% der DMP-Teilnehmer mit „bis zu 4 Jahren“ (Nichtteilnehmer: 22,0\%), 36,0\% mit „5 bis 10 Jahren“ (Nichtteilnehmer: 40,0\%), 32,4\% mit "11 bis 20 Jahren" (Nichtteilnehmer: 25,6\%) und $14,7 \%$ mit „mehr als 20 Jahren“ (Nichtteilnehmer: 11,3\%). Damit ergibt sich alters- und geschlechtsstandardisiert signifikant häufiger eine längere Dauer der Erkrankung. Die Krankheitsdauer korreliert positiv mit der Häufigkeit von Begleit- und Folgeerkrankungen und kann daher als aussagekräftig im Hinblick auf die Krankheitsschwere angesehen werden ${ }^{15}$ (s. Grafik 1).

Die Frage nach der subjektiven Schwere des Diabetes bestätigt dies: 28,5\% der DMP-Teilnehmer fühlen sich „weniger schwer bis nicht schwer" erkrankt, während dieser Anteil bei den Nichtteilnehmern mit 34,3\% signifikant höher liegt.
Graphik 1: Wie lange sind Sie bereits an Diabetes mellitus Typ 2 erkrankt? Versichertenanteile mit Erkrankungsdauer in Jahren

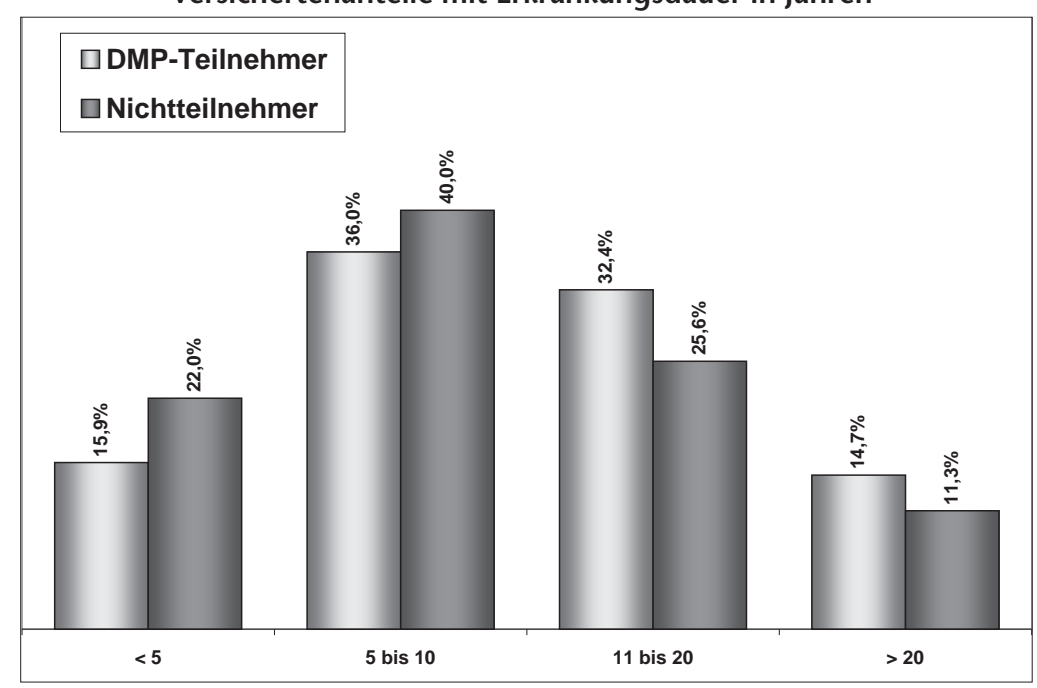

Quelle: BARMER 
Graphik 2: Wie wird Ihr Diabetes behandelt?

Versichertenanteile mit entsprechenden Angaben zur Basistherapie (Diät, Bewegung) und antidiabetischen Medikation.

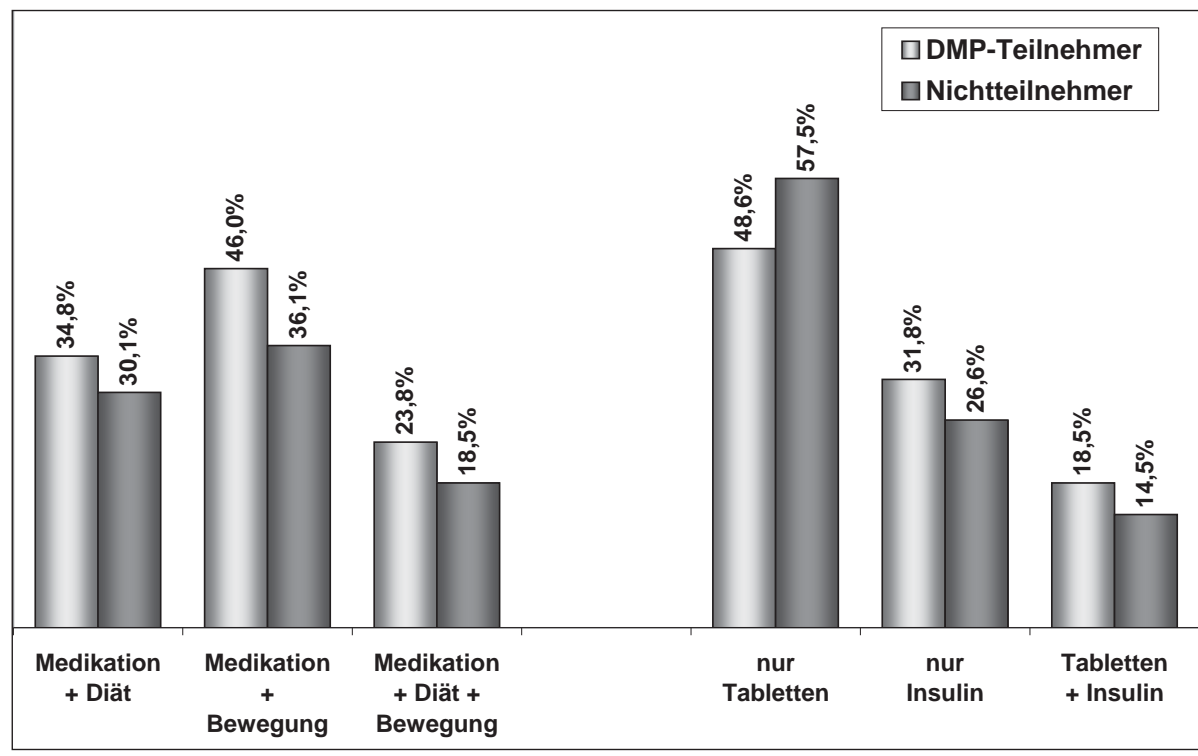

Quelle: BARMER

Auch der Anteil insulinbehandelter Patienten kann als Indikator für die Krankheitsschwere gewertet werden ${ }^{16}$ Hierbei zeigt sich, dass 50,3\% der Diabetes-Patienten im DMP mit Insulin behandelt werden, während nur 41,1\% der Nicht-DMP-Patienten insulinpflichtig sind (s. Grafik 2).

Hinsichtlich der Form der Diabetestherapie zeigt sich unterdessen ein weiteres interessantes Ergebnis (s. Grafik 2): DMP-Teilnehmer geben signifikant häufiger an, dass ihre Behandlung neben der Medikation auch Diät und/oder Bewegung umfasst. Trotz der höheren diabetesspezifischen Krankheitsschwere wird bei DMP-Teilnehmern somit die elementare Basistherapie des Diabetes Typ 2 häufiger angenommen und umgesetzt als von Nichtteilnehmern.

Schließlich zeigt auch die Auflistung von diabetesassoziierten Begleit- und Folgeerkrankungen sowie sonstiger Krankheiten insgesamt eine deutlich größere Häufung bei den DMP-Teilnehmern.

Bezüglich der typischen Begleit und Folgeerkrankungen des Diabetes mellitus gaben die DMP-Teilnehmer häufiger an, von Risikofaktoren für makroangiopathische Folgeerkrankungen und Komplikationen betroffen $\mathrm{zu}$ sein. Signifikante Unterschiede liegen beim Bluthochdruck $(67,8 \%$ vs. 61,2\%) und bei Männern zusätzlich bei der Koronaren Herzkrankheit vor
(13,7\% vs. $10,7 \%)$. Bei den zusätzlichen Risikofaktoren, die zu Minorund Majoramputationen der unteren Extremitäten führen können, (Neuropathie und Diabetisches Fußsyndrom) finden sich keine signifikanten Unterschiede (s Grafik 3).

Die Angaben zu den weiteren (nicht diabetestypischen) Erkrankungen weisen darauf hin, dass auch die Gesamtmorbidität unter den Teilnehmern höher einzustufen ist als bei Nichtteilnehmern. Bemerkenswert ist an dieser Stelle, dass die von DMP-Teilnehmern häufiger angegebenen Erkrankungen des Bewegungsapparates offensichtlich keinen Grund für eine seltenere Teilnahme am DMP darstellen.

Zusammenfassend sprechen diese Befragungsergebnisse somit keinesfalls für eine Selektion „risikoärmerer" Versicherter bei den DMP-Teilnehmern, sondern für das Gegenteil. Das Diabetesstadium wird von bei Teilnehmern signifikant seltener als „wenig schwer bis nicht schwer“ eingestuft, und die Erkrankung wird häufiger mit Insulin behandelt. Da die Teilnehmer durchschnittlich bereits deutlich länger vom Diabetes mellitus betroffen sind, sind diese Angaben auch nicht überraschend, sondern belegen folgerichtig eine höhere „Diabetesmorbidität" unter den DMP-Teilnehmern. DMP-Teilnehmer haben zudem häufiger diabetesassoziierte Begleiterkrankungen sowie sonstige Krankheiten.

Graphik 3: Welche der folgenden Krankheiten bzw. Beschwerden hatten Sie in den letzten 12 Monaten? Versichertenanteile mit entsprechenden Angaben

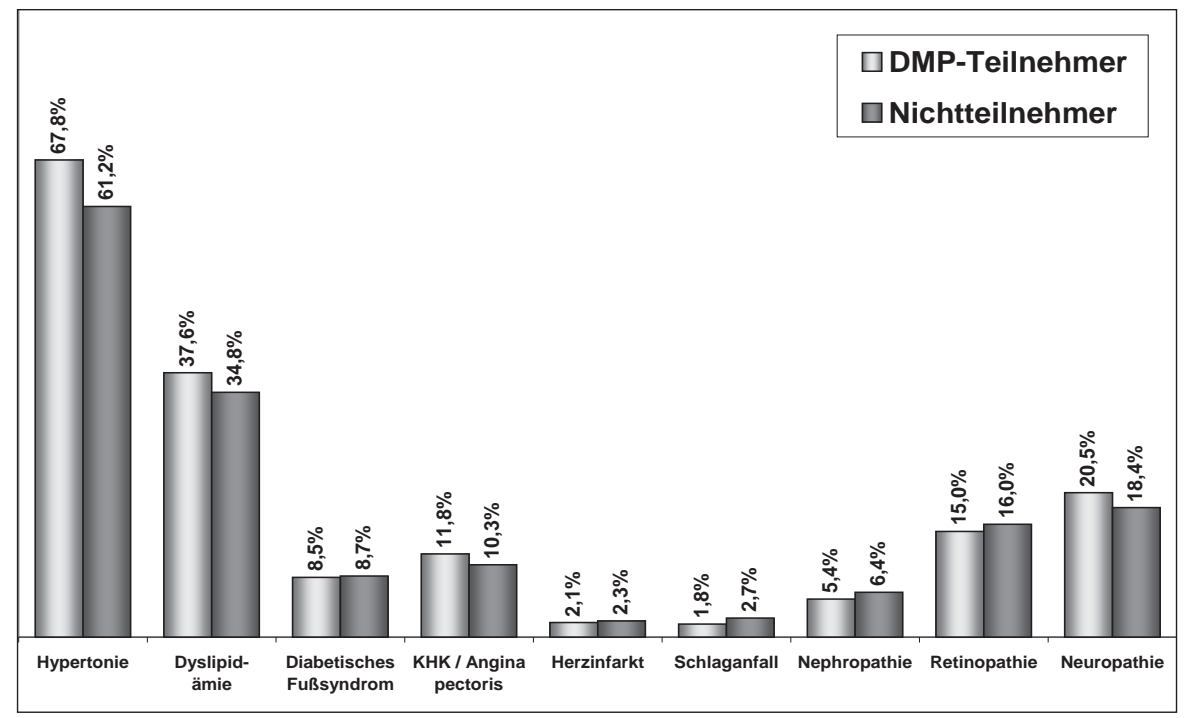

Quelle: BARMER 


\subsection{Sozialstatus}

Aus der Befragung können Aussagen über den Bildungsstand der Befragten abgeleitet werden. Da der Faktor Bildung als wesentlicher Indikator für den Sozialstatus gilt, lassen sich somit Rückschlüsse ziehen, ob im Rahmen der DMP möglicherweise ein sozial selektives Teilnahmeverhalten stattfindet. Eine solche Selektivität wird insbesondere bei der Inanspruchnahme von Maßnahmen der primären Prävention seit vielen Jahren diskutiert. ${ }^{17}$

Bei den Befragten zeigt sich ein signifikant höherer Anteil Personen mit Realschulabschluss oder (Fach)-Hochschulreife unter den DMP-Teilnehmern (48,9\% vs. 43,6\%), wohingegen der Anteil Personen mit Volks- oder Hauptschulabschluss unter den Nichtteilnehmern signifikant höher ist (50,7\% vs. 46,1\%). Im Bereich der Berufsausbildung bestätigt das Bild diese Tendenz. Der Anteil Personen ohne Berufsausbildung ist unter den Nichtteilnehmern signifikant höher (6,3\% vs. 3,6\%).

Hingegen ist unter den Teilnehmern der Anteil mit Fachhochschulausbildung (Ingenieur) signifikant höher (11,9\% vs. 7,8\%).

Insgesamt kann somit ein moderater Selektionseffekt beim Bildungs- und Ausbildungsstand innerhalb der DMP festgestellt werden.

\subsection{Informationsstand}

Im Rahmen der Erhebung wurden zahlreiche Fragen zum Informationsstand und zum Informationsverhalten der Versicherten gestellt, die durchweg einen besseren Informationsstand und ein aktiveres Informationsverhalten bei DMP-Teilnehmern zeigen ${ }^{2}$. Besonders deutlich wird dieser Unterschied bei der Frage nach der Kenntnis des HbA1c-Wertes. Hierbei handelt es sich um einen zentralen Parameter zur Langzeiteinstellung des Blutzuckerwertes. Die Kenntnis dieses Wertes wird gemeinhin als Ausdruck für ein erfolgreiches Patientenselbstmanagement angesehen. ${ }^{18}$

Während 79,8\% der befragten DMPTeilnehmer angeben, ihren HbA1c-Wert zu kennen, liegt dieser Anteil bei den Nichtteilnehmern mit 55,8\% geradezu drastisch niedriger. Dieser große Unterschied kann nicht allein mit dem allgemein höheren Bildungsstand erklärt werden. Vielmehr ist zu erwarten, dass das Informationsverhalten, aber auch das Informationsangebot bei diesen Patienten deutlich günstiger ist. So ist der Anteil Patienten, die an einer Gruppenschulung teilgenommen haben, innerhalb der DMP deutlich höher ${ }^{2}$. Dieser Umstand könnte auch erklären, warum DMP-Teilnehmer signifikant häufiger angeben, dass ihre Behandlung neben der Medikation auch Diät und/oder Be-

Quelle: BARMER wegung umfasst (s. Graphik 2). Dies alles deutet auf ein tiefer greifendes Verständnis der Krankheitszusammenhänge hin, so dass die elementare Basistherapie und das Selbstmanagement des Diabetes Typ 2 von Teilnehmern häufiger angenommen und umgesetzt wird als von Nichtteilnehmern.

Auch hier ist ein Selektionseffekt insoweit denkbar, als Patienten mit einem höheren Problembewußtsein für ihre Erkrankung und einem entsprechend aktiveren Informationsverhalten womöglich eher eine DMP-Teilnahme wählen. Dieser Effekt dürfte sich aber im Langzeitverlauf mit einem tatsächlichen Impuls durch das Angebot der DMP mischen. Letztlich folgen zunehmend mehr Patienten dem „guten Beispiel“ und die bereits aktiv Teilnehmenden werden in ihrem Verhalten bestätigt. Im Rahmen eines Programmes, dass explizit die aktivere Beteiligung und Information der Patienten zum Ziel hat und auch entsprechend kommuniziert wird, muss eine solche Wirkung schließlich als naheliegend erachtet werden.

\subsection{Versorgungsstruktur}

Ob die aufgezeigten Unterschiede auch mit einer unterschiedlichen Versorgungsstruktur innerhalb und außerhalb der DMP einhergehen, kann im Rahmen dieser Patientenbefragung u.a. daran abgelesen werden, wo die Patienten in den letzten 12 Monaten wegen ihres Diabetes behandelt wurden.

Der Anteil der Patienten, die bei einem nicht diabetologisch spezialisierten Allgemeinarzt, praktischen Arzt oder Internisten behandelt wurden, liegt mit 77,1\% bei den DMP-Teilnehmern etwas niedriger als bei den Nichtteilnehmern (83,0\%). Dagegen ist der Anteil der bei einem Diabetologen behandelten Patienten mit 39,8\% für DMP-Teilnehmer beinahe doppelt so hoch (Nichtteilnehmer: 20,8\%). Somit werden Patienten im DMP häu-

Graphik 4: Wo waren Sie in den letzten 12 Monaten wegen Ihres Diabetes in Behandlung?

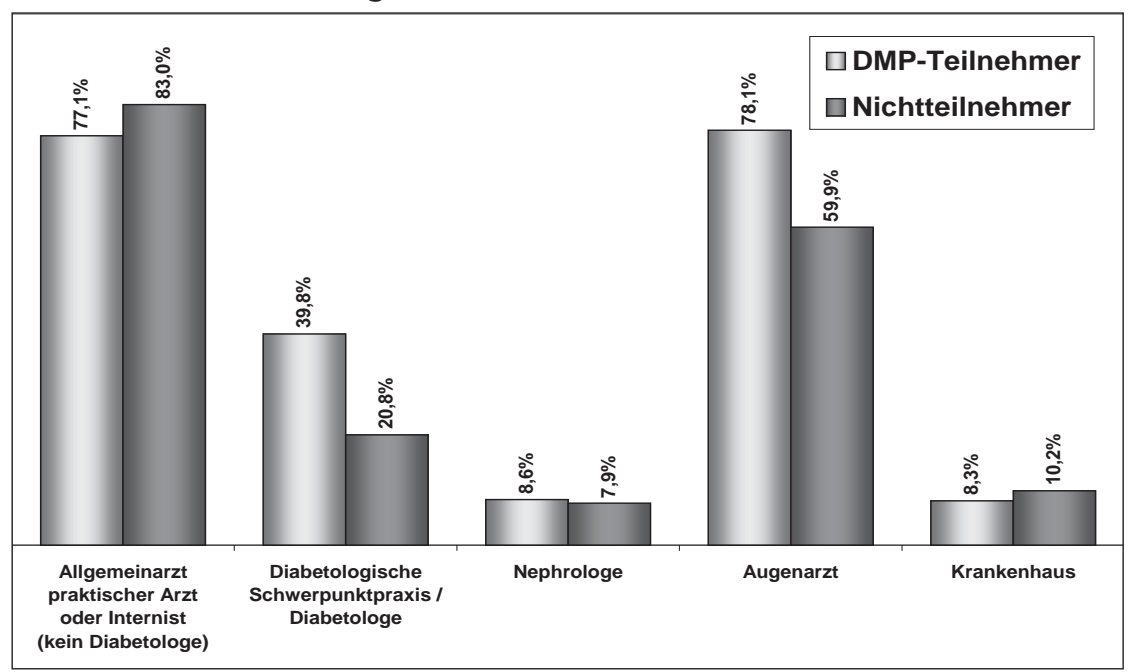


figer zusätzlich zum Hausarzt von einer diabetologischen Schwerpunktpraxis betreut, als Diabetiker außerhalb der DMP (siehe Grafik 4).

Im welchem Maß die längere Erkrankungsdauer einen Grund für die deutlich häufigere (Mit-) Behandlung durch eine Schwerpunktpraxis darstellt, kann nur schwer abgeschätzt werden. Für den auffälligen Unterschied in der Behandlung von Teilnehmern und Nichtteilnehmern sind jedoch grundsätzlich zwei Erklärungsansätze denkbar: Entweder führt die DMP-Teilnahme bei Ärzten und Patienten dazu, dass häufiger vertragskonform an die zweite Versorgungsebene überwiesen wird. Auch die engere Kooperation, z.B. bei der Patientenschulung ist hierbei im Sinne des DMP denkbar. Oder es findet auch hier ein Selektionseffekt statt, indem vor allem Diabetologische Schwerpunktpraxen und Hausärzte, die bereits enger mit Diabetologen kooperieren, häufiger an den DMP teilnehmen und ihre Patienten einschreiben.

Die Tatsache, dass DMP-Teilnehmer sehr viel häufiger auch eine augenärztliche Behandlung innerhalb der letzten 12 Monate angaben (78,1\% vs. 59,9\%), unterstreicht in diesem Zusammenhang erneut die deutlichen Unterschiede in der Prozessqualität. Bemerkenswert ist an dieser Stelle auch, dass Teilnehmer signifikant seltener wegen ihres Diabetes im Krankenhaus behandelt werden mussten. Auch dieses Ergebnis spricht dafür, dass DMPTeilnehmer seltener Komplikationen und Entgleisungen erleiden, was $u$. a. auf eine optimiert ambulante Therapie zurückgeführt werden könnte.

Während es im Kontext der oben dargestellten Leistungsdatenanalyse noch zumindest fraglich erschien, ob die besseren Ergebnisse hinsichtlich Prozess- und Ergebnisqualität auch durch Selektionseffekte im Bereich der „Morbidität" beeinflusst sind, zeigt die Versichertenbefragung hier ein deutliches Bild: Trotz einer auffällig höheren Krankheitsschwere und zahlreicherer Begleit-erkrankungen erreichen DMP-Teilnehmer anscheinend bessere Behandlungsergebnisse, die im übrigen mit einer höheren Behandlungszufriedenheit einhergehen². Maßgebliche Erklärungsansätze hierfür liegen im Informationsstand der Versicherten sowie in der unterschiedlichen Versorgungsstruktur, insbesondere im deutlich höheren Anteil der von einer diabetologischen Schwerpunktpraxis mitbehandelten Patienten im DMP.

\section{Zusammenfassung und Ausblick}

Die andauernde Diskussion über den Nutzen der DMP und dessen Nachweisbarkeit hat durch die dargestellten Untersuchungen wichtige neue Impulse erhalten. Während bisherige Qualitätsberichte und die gesetzlich vorgeschriebene Programmevaluation mangels Vergleichsgruppen bislang keine überzeugenden Resultate über die spezifische Versorgungsverbesserung bei DMP-Teilnehmern erbracht haben (und wohl auch nicht erbringen können), zeigt der Vergleich von DMP-Teilnehmern und Nichtteilnehmern systematische Versorgungsunterschiede auf. Für beide dargestellten Untersuchungen - eine Analyse von Leistungsdaten sowie eine Versichertenbefragung - gilt hierbei, dass vielfältige Einflussfaktoren und mögliche Selektionseffekte (wie in der Versorgungsforschung generell) besonders betrachtet und diskutiert werden müssen.

Die Analyse der Leistungsdaten von rund 160.000 Diabetikern zeigt, dass DMP-Teilnehmer insbesondere wegen schwerer Komplikationen seltener im Krankenhaus behandelt werden mussten und dass die Schlaganfall- und Amputationsraten bei dieser Gruppe signifikant niedriger lagen. Dagegen waren DMP-Teilnehmer signifikant häufiger bei einer augenärztlichen Untersuchung und wurden häufiger mit Medikamenten versorgt, die das Risiko für schwerwiegende Komplikationen bei Diabetikern senken

Der Unterschied bei der Arzneimittelversorgung ist im Hinblick auf mögliche Selektionseffekte bei der Morbidität der Versicherten besonders bemerkenswert: Daraus kann entweder der Schluss gezogen werden, dass die DMP-Teilnehmer häufiger Begleiterkrankungen haben, aber dennoch bessere Behandlungsergebnisse im Sinne der geringeren Schlaganfall- und Amputationsraten aufweisen. Oder die intensivere Arzneimittelversorgung ist unabhängig von der Morbidität ein weiterer Indikator für die bessere Behandlung bei DMP-Patienten.

Unterdessen zeigen die Ergebnisse der vergleichenden Versichertenbefragung, dass eingeschriebene Versicherte signifikant länger an Diabetes mellitus erkrankt sind, Ihre Krankheit schwerer einschätzen und häufiger insulinpflichtig sind. Zudem weisen DMP-Teilnehmer häufiger diabetesassoziierte und sonstige Begleiterkrankungen auf. Damit liegen deutliche Hinweise vor, dass die Gesamtmorbidität bei Diabetikern, die im DMP eingeschrieben sind, höher ist als bei nichteingeschriebenen Diabetikern. Dennoch zeigen sich bei DMP-Teilnehmern bessere Ergebnisse der Prozess- und Ergebnisqualität, die nicht zuletzt mit einer höheren Patientenzufriedenheit einhergehen.

Wie sind diese Zusammenhänge erklärbar? Ein häufig vorgebrachter Einwand könnte in der sozialen Selektivität des Einschreibeverhaltens liegen. Die Ergebnisse der Versichertenbefragung zeigen einen geringen Unterschied im Bildungsniveau. Ein erheblich größerer Unterschied wird jedoch beim Informationsverhalten und beim Informationsstand der Versicherten sichtbar. So ist der Anteil der Patienten, die ihren HbA1C-Wert kennen, in der Gruppe der DMP-Teilnehmer über ein Drittel höher als in der Gruppe der Nichtteilnehmer. Dies korreliert mit einem deutlich höheren Anteil an Versicherten, die eine Schulung absolviert haben und nicht zuletzt mit einem höheren Anteil Patienten, die neben der medikamentösen Therapie eine Basistherapie in Form von Bewegung und Diät umsetzen. Neben dem tendenziell höheren Bildungsniveau müssen somit weitere wichtige Faktoren zu diesen deutlichen Unterschieden geführt haben.

Ein maßgeblicher Einflussfaktor ist die Versorgungsstruktur. Während rund 80 Prozent aller Befragten im 
letzten Jahr hausärztlich behandelt wurden, ist die Zahl der Patienten, die zudem durch eine diabetologische Schwerpunktpraxis behandelt wurden, bei DMP-Teilnehmern fast doppelt so hoch. Dieser Strukturunterschied könnte der Schlüssel für eine Vielzahl der aufgezeigten Phänomene sein. Patienten, die am DMP teilnehmen, sind besser informiert, häufiger geschult, werden öfter zur augenärztlichen Vorsorge überwiesen und seltener im Krankenhaus behandelt. Zudem geben diese Patienten häufiger an, dass ihr Arzt Therapieziele mit ihnen vereinbart hat, dass die jährlich notwendige Fußinspektion durchgeführt wurde und dass sie sich durch ihren Arzt und die Helferin gut informiert fühlen ${ }^{2}$. Die in der Leistungsdatenauswertung festgestellten Ergebnisse - häufigere protektive Medikation bei geringeren Ereignisraten an Schlaganfällen und Amputationen - fügt sich nahtlos in dieses Bild.

Dennoch bleibt die berühmte Frage „nach der Henne und dem Ei": Zu welchen Anteilen sind die festgestellten Strukturunterschiede Ergebnis der DMP-Einschreibung bzw. die Voraussetzung, dass es überhaupt zu einer Einschreibung kommen konnte? Aus der Praxis ist weithin bekannt, dass neben der freiwilligen Entscheidung der Patienten für oder gegen eine DMP-Teilnahme stets auch die Grundeinstellung des jeweils behandelnden Arztes ausschlaggebend ist. Die Polarisierung zwischen „DMPVerweigerern“ und „DMP-Fürsprechern" hat in der Vergangenheit dazu geführt, dass zumindest der Vergleich von am DMP teilnehmenden und nichtteilnehmenden Versicherten zu einem hohen Prozentsatz auch Hinweise für einen Vergleich von am DMP teilnehmenden und nichtteilnehmenden Ärzten lieferte. Insoweit ist zumindest partiell davon auszugehen, dass ein Selektionseffekt insoweit stattgefunden hat, als vor allem Diabetologische Schwerpunktpraxen und Hausärzte, die bereits enger mit Diabetologen kooperieren, häufiger an den DMP teilnehmen und ihre Patienten einschreiben.

Ein relevanter Teil der beschriebenen Effekte dürfte aber auch durch die Wirkung der DMP selbst auf das Verhalten bei Ärzten und Patienten entstanden sein. So ist anzunehmen, dass innerhalb der DMP nicht nur häufiger vertragskonform eine regelmäßige Augen- und Fußuntersuchung veranlasst bzw. durchgeführt wird, sondern auch häufiger vertragskonform an die zweite Versorgungsebene überwiesen wird als außerhalb der DMP-Verträge. Auch das häufigere Schulungsangebot kann ein Resultat der DMP-bedingten Veränderungsprozesse gewesen sein. Vielerorts wurden Schulungsgemeinschaften gegründet, die wiederum zu einer intensivierten Kooperation zwischen Hausärzten und Diabetologen geführt haben. Schließlich dürfte auch die anhaltende Diskussion über Leitlinieninhalte seit 2002 bei denjenigen Ärztinnen und Ärzten häufiger Gehör gefunden haben, die über eine grundsätzliche Affinität zu strukturierten Behandlungsprogrammen verfügen.

Auch patientenseitig ist anzunehmen, dass sich initiale Selektionseffekte von Patienten mit einem höheren Problembewußtsein für ihre Erkrankung und einem entspre- chend aktiveren Informationsverhalten innerhalb der DMP bei zunehmender Programmdauer mit tatsächlichen Impulsen durch das Angebot der DMP mischen. Letztlich folgen zunehmend mehr Patienten dem "guten Beispiel“ und die bereits aktiv teilnehmenden Versicherten werden in ihrem Verhalten bestätigt. Diese Wirkung in Bezug auf die aktive Beteiligung und Information der Patienten ist ausdrückliches Ziel aller DMP-Verträge. Allein die Ankündigung und Erläuterung dieser Programminhalte könnte mancherorts Ausgangspunkt für Veränderungen gewesen sein. Nicht zuletzt haben auch die umfassenden und speziellen Informationsmaterialien, die eigens von den Krankenkassen den DMP-Teilnehmern zur Verfügung gestellt werden, zusätzlich zum verbesserten Informationsstand beigetragen.

All dies muss bis zu einem gewissen Grad offen und spekulativ bleiben. Für die Zukunft können Follow upUntersuchungen sicherlich noch mehr Klarheit über Veränderungen im Programmverlauf erbringen. In diesem Punkt zeigen die Qualitätsberichte und gesetzliche Programmevaluation schon jetzt einen positiven Trend, der aber zukünftig im Vergleich zur Regelversorgung außerhalb der DMP validiert werden sollte.

Klar ist aber schon jetzt, dass es einen systematischen Unterschied in der Versorgungsqualität bei DMP-Teilnehmern und Nichtteilnehmern gibt. Insoweit kann heute sowohl Ärzten als auch Patienten nur geraten werden, dem Positivbeispiel der strukturierten Behandlung zu folgen. Für die Gesamtbevölkerung lassen sich dabei die Potenziale zur Verbesserung der Versorgung besser als bisher abschätzen.

Dieser systematische Unterschied in der Versorgungsqualität zeigt sich beinahe auf allen Ebenen und liefert mithin sogar Grund zur Annahme, dass DMP-Versicherte kurz-, mittel- und langfristig deutlich wirtschaftlicher versorgt werden. Die selteneren Krankenhausaufenthalte und Komplikationsraten lassen erhebliche Ersparnisse erwarten. Bei der Bemessung der zukünftigen Beitragsbedarfe für Diabetiker im Rahmen des Morbi-RSA könnte die aus den Daten erkennbare höhere Morbidität der DMP-Teilnehmer (u.a. aufgezeigt anhand der überdurchschnittlich häufigen spezifischen Arzneimittelverordnungen), zu einer mindestens ebenso hohen Mittelzuweisung führen wie bei Nichtteilnehmern. Dies gilt natürlich nur dann, wenn die hier herausgearbeiteten Morbiditätsmerkmale im Morbi-RSA für Diabetiker Berücksichtigung finden. Damit wären die Voraussetzungen geschaffen, um DMP dauerhaft, auch ohne „quasi-Subvention“ über den RSA, als Steuerungsinstrument in der GKV zu etablieren.

Neben dem einzelwirtschaftlichen Interesse der Krankenkasse gibt es aber im Falle der DMP - auch angesichts der aufgezeigten Ergebnisse - ein gesamtgesellschaftliches und gesundheitspolitisches Interesse. Rund die Hälfte aller Patienten mit Diabetes mellitus in Deutschland sind mittlerweile in ein DMP eingeschrieben und profitieren nachweislich von dieser Versorgungsform mit all ihren Begleitfaktoren. Es wäre vor diesem Hintergrund 
aus Sicht des Gesetzgebers nahezu undenkbar, etwa ausgelöst von einem kurzfristigen Kostendruck bei einzelnen Krankenkassen ab 2009 einen „radikalen Rückbau“ entsprechender Angebote für chronisch Kranke einfach „hinzunehmen“. Wie bei anderer Gelegenheit in der jüngsten Reformvergangenheit gezeigt, könnte die Politik rechtzeitig den Rahmen setzen, dass ein Nichtangebot von DMP - etwa als „innovatives Mittel der Risikoselektion" - nicht möglich oder zumindest hinreichend unattraktiv wäre.

\section{Fußnoten}

1 Ullrich W., Marschall U., Graf C.: Versorgungsmerkmale des Diabetes mellitus in Disease

Management-Programmen. Ein Vergleich von in die DMP eingeschriebenen und

nichteingeschriebenen Versicherten mit Diabetes. Diabetes, Stoffwechsel und Herz 6/2007,

S. $407 \mathrm{ff}$.

2 Elkeles et al., in diesem Heft

3 Nagel H., Behring Th., Scherbaum W. et al.: Implementing Disease Management Programms for typ two Diabetes in Germany. Managed Care 11/06, S. 50 ff.

4 Graf C., Disease Management Programme und Integrierte Versorgung. Gesundheits- und

Sozialpolitik 5-6/2006, S. 42 ff.

5 beispielhaft: Ärztezeitung v. 01.12.2006: DMP-Erstdokumentationen werden zur Minutensache

6 IGES: Voraussetzungen für ein effektives und effizientes Disease Management für Typ-2-Diabetiker

und seine adäquate Finanzierung im Rahmen der gesetzlichen Krankenversicherung. Berlin 2003

7 Wagner E.H., Austin B.T., Davis C. et al.: Improving chronic illness care: Translating evidence into action. Health Affairs 2001; 20 (6), S. 64 ff. sowie Ofman J., Badamgarav E. et al.: Does disease management improve clinical and economic outcomes in patients with chronic diseases? A systematic review. Am J Med 2004; 117, S. $182 \mathrm{ff}$.

8 exemplarisch s. Nordrheinische gemeinsame Einrichtung Disease Management Programme GBR:

Qualitätssicherungsbericht 2005. Disease Management Programme in Nordrhein.

zusammenfassend s. Graf, C. a.a.O.

9 Die noch weiter gehende Forderung nach randomisierten, kontrollierten Studien (RCT) im DMP führt hingegen nach Ansicht der Autoren am Wesen und Ziel der Versorgungsforschung - der die DMPEvaluation konzeptionell zuzuordnen ist - vorbei. Ungeachtet der potenziellen Wirksamkeit unter „Laborbedingungen“ interessiert in einem so komplexen Setting wie der strukturierten Chronikerversorgung die „real life-Betrachtung“. Dabei sind verschiedene UrsacheWirkungs-Beziehungen möglich, die es im zweiten Schritt ggf. näher zu untersuchen gilt. (vgl. Pfaff H. (2003): Versorgungsforschung Begriffsbestimmung, Gegenstand und Aufgaben. In: Pfaff, Schrappe, Lauterbach u.a. [Hrsg.]: Gesundheitsversorgung und Disease Management. Verlag Hans Huber, Berlin, S. 13 ff.)

10 Die Einschreibepraxis im DMP zeigt im übrigen eine deutliche Segmentierung zwischen Ärzten, deren Patienten nahezu vollständig am DMP teilnehmen und Ärzten, die keine oder nur sehr wenige Patienten eingeschrieben haben. Der oftmals vorgebrachte Einwand, dass ein Patient bei dem gleichen Arzt wohl gleich behandelt würde, unabhängig davon ob er ins DMP eingeschrieben ist oder nicht, geht somit an der Realität meistens vorbei. Wenn ein Patient am DMP teilnimmt, war die Wahl seines Arztes hierfür oftmals bereits eine entscheidende Bedingung.

11 Häussler B., Wille, E., Wasem J., Storz Ph.: Diabetiker im Disease Management. Erste Erkenntnisse über die Wirkung der Disease Management Programme in der gesetzlichen Krankenversicherung. Gesundheits- und Sozialpolitik 9-10/2005, S. 23 ff.

12 Gemeinsame Therapieziele der DMP Diabetes mellitus Typ 1 und 2 sind eine Verbesserung der von einem Diabetes mellitus beeinträchtigten Lebensqualität und Vermeidung diabetesbedingter und assoziierter Folgeschäden, u.a:

- Reduktion des erhöhten Risikos für kardiale, zerebrovaskuläre und sonstige makroangiopathische Morbidität (Herzinfarkt, Schlaganfall), u.a. beeinflusst durch evidenzbasierte Medikation.

- Vermeidung der mikrovaskulären Folgekomplikationen (insbesondere Retinopathie mit schwerer Sehbehinderung oder Erblindung, Niereninsuffizienz mit der Notwendigkeit einer Nierenersatztherapie), u.a. beeinflusst durch regelmäßige augenärztliche Kontrollen und evidenzbasierte Medikation

- Vermeidung des diabetischen Fußsyndroms mit neuro-, angio- und/ oder osteo- arthropathischen Läsionen und von Amputationen, u.a. beeinflusst durch regelmäßige Inspektion der Füße. (vgl. RSAV, Anl. 1 und 7)

13 Reschke P, Sehlen S, Schiffhorst G et al.(2005): Klassifikationsmodelle für Versicherte im

Risikostrukturausgleich. Bonn: Bundesministerium für Gesundheit und soziale Sicherung, Referat Information, Publikation, Redaktion

14 Nuber G., Hillenbrand H., DMP-Teilnehmern geht's besser. Diabetesjournal 4/2006, S. $20 \mathrm{ff}$.

15 Böhler et. al., Versorgungsqualität des Typ-2-Diabetes- erste Ergebnisse aus der DETECT-Studie. Diabetes, Stoffwechsel und Herz, Suppl $1 / 2006$

16 Pittrow D et al., Diabetes mellitus in der Hausarztpraxis, Med Klein 2006; 101:635-44 (Nr. 8)

17 Robert Koch Institut (Hrsg)2006, Gesundheit in Deutschland. Gesundheitsberichterstattung des Bundes. Robert Koch-Institut, Berlin

18 Lippmann-Grob, B., Hillenbrand, H., Kolonko, B., Vogelmann, M., Diabetikerversorgung in Baden Württemberg. In: Diabetes, Stoffwechsel und Herz, 1/2006, S. 21 ff. 